\title{
Design of Residential Ecological Balcony in Northwest Area
}

\author{
Fangli $\mathrm{Li}^{1, \mathrm{a}}$ \\ ${ }^{1}$ Lanzhou Jiaotong University, Lanzhou, Gansu, China, 730070 \\ a email
}

Keywords: Sediment Control Ventilation, Balcony Structure, Northwest Area

\begin{abstract}
Currently, there are frequent sandstorms in Northwest China, to reduce the impact of extreme weather on the indoor living environment and respond to doors and windows sealed due to occlusion of the indoor environment, we must transform the balcony design and balcony design must meet ecological ventilation, dust and other isolated Features. How to create a structure in the form of green planting both a highly efficient anti-dust and to achieve good indoor natural ventilation systems ecology balcony is the purpose of this paper.
\end{abstract}

\section{Introduction}

Sand storm has been an important factor affecting the Northwest high quality living environment. Northwest dried whole climate, with large temperature difference between day and night, easy to form windy weather. Such strong winds and more mixed with sand and burning coal for heating in winter release of cinder, harmful to the environment. Especially in the construction of the leeward area, the wind is often in the back of the building to form a vortex area, is not conducive to the renewal and air circulation. Dust weather hazards not only in the destruction of vegetation, buried farmland, impact on traffic, air pollution and dust weather led to the sharp increase in suspended particulate matter in the air, easy to induce a variety of respiratory diseases. Response Northwest bad weather, we at the same time gradually improving external environment, but also must pay attention to the ecological nature of the indoor environment. A day in about $70 \%$ to $90 \%$ of their time is spent indoors, indoor air quality and human mental health, physical health are closely related. Balcony opening position as an important building is not a long time to draw attention to the weak link. Whether it is a key part of the summer heat or winter insulation, indoor and outdoor balcony space as a transitional contact affect buildings are environmentally friendly, and the location of the balcony with the "variable, adjustable," the many possibilities, so eco-Northwest balcony design is a means to alleviate the impact of bad weather on the indoor environment.

\section{Northwest Situation of Balcony Designed and Its Inadequate}

The balcony is the outdoor terrace bar dry construction and house building Central evolution is from space and space is the extension of home life and has some privacy.

Northwest region due to the need for anti-sandstorm, balcony general use of double windows, double windows to resist the sandstorm disasters better. But when the dust storms of longer duration, long time no ventilation can lead to living space air quality. Plus sandstorms often occur in spring and summer, no ventilation can cause indoor hot, affecting people's health, so the double glazing is only suitable for short-term local dust storms occur, but not for a longer duration of dust storms in northwest China.

Due to the cold insulation, Northwest region energy-saving insulation and anti-sand needs, widely used in semi-enclosed balcony and double window form. Semi-enclosed balcony has a good form of insulation in the winter, but the ventilation disadvantage.

Northwest large temperature difference between day and night, year-round daily temperature range is $12{ }^{\circ} \mathrm{C}-16{ }^{\circ} \mathrm{C}$, higher than the eastern coastal plain region more than doubled, up to the maximum daily range $25{ }^{\circ} \mathrm{C}-34{ }^{\circ} \mathrm{C}$. Northwest and long hours of sunshine, the weather conditions on the building insulation and insulation have a great challenge. 90 years ago in the 21st century, northwest balcony use of semi-enclosed balcony, has a thermal insulation effect. The late 1990s, 
with the crazy real estate development industry, urban architecture become standardized, the new multi-storey residential balcony on the issue of whether the closure, real estate developers, has played a leading role, to focus only on short-term economic benefits, can not be considered "standard" consumption, climatic conditions emerged balcony. Every City-style balcony design, there is no local characteristics northwest, contact balcony as indoor and outdoor space, as the flow of heat to convert its multiple functions disappear.

The statistics, a five-story residential buildings, walls and balconies green area of the residential area of about three or four times, it can be said the balcony greening urban greening indispensable part. Balcony green northwest generally no particular design is more simple planting pot. In the choice of plants without considering the temperature, they live in large sand and other climate characteristics. Meanwhile, the plant regulation of indoor air humidity to almost zero and compared to other regions, the greater Northwest balcony green design has difficulty.

\section{Study of Northwest Ecological Balcony Design}

Northwest monsoon accordance with the principles and characteristics of building construction features and indoor ventilation should be low prices, and in the side of the balcony window is provided below the air inlet and exhaust port opening in the top of the opposing side, the vent should be set to carve close It forms, in order to ensure the flexibility of ventilation. Balcony drives natural ventilation in indoor air circulation, so as to achieve a natural circulation of air in winter indoor and outdoor purposes.

After the principle according to the indoor ventilation, air and set the path where the balcony airflow exchange, the next question to be considered is how to solve the sandstorm weather, the indoor air cleanliness issues. To resolve sandstorm weather ventilation problems balcony of the original structure in the form of exterior windows in here do not apply, we need to change the existing ordinary window constructed at the same time be able to guarantee the isolation of sand into the room through the air apertures. Sand filtering blinds, not only can prevent dust into the room, and can effectively achieve indoor natural ventilation. Blinds consists of two parts, one part is tilted in the Z-shaped louvers, the other part is affixed to the inside louver filter, electrostatic adsorption of the fiber network, activated carbon fiber filters. Use the windshield tilted block off the larger particle diameter of sand to change the air flow direction. Use of environmentally friendly fiber filter material, the use of wind velocity dust storms come, so that the air flow through the filter into the interior, the process of non-polluting, high efficiency. This dust weather of Northwest China to improve indoor living conditions has a strong practical.

Various forms of regular balcony will bring lively residential building facade beauty. Hot summer and cold winter area multi-storey residential convex balcony railing balcony building design to follow the general rules of design and it can be used in combination with arc straight punch, the actual situation changes and other practices.

Rich and diverse shapes tailgate variable shade way organic combination, will create architectural forms in line with balcony northwest climatic features, designed to provide the possibility based on rational "core" variety.

In order to take into account the balcony warm and airy balcony in the form of a balcony constructed northwest can form under the window walls, windows and wall binding. Balcony window with double glass material, the use of the balcony wall insulation materials, and set the radiator vents near the balcony at least one group.

The main features of adaptive planting reflected in the choice of plants that grow in the area to choose suitable varieties of planting. Compared to other regions, the common mode is greater than green balcony personality, in accordance with the following characteristics Northwest climate to introduce balcony Greening Design.

Balcony orientation, height and shape are very different depending on the design. In addition to placing potted green balcony on the balcony outside the window, the main way of various types of outdoor planting groove civil synchronous built to meet the beautiful Northwest summer and autumn heat demand; more concentrated in the winter greening balcony inside, it can be 
three-dimensional green on the top, side and other corners of the location of various types of hanging pots.

Greening the balcony can be reduced indoor and outdoor temperature in summer and autumn, winter indoor humidity increase, the balcony is essential ecological design.

\section{Conclusion}

Good building respiratory system is critical for ecological building design, for different regions in terms of construction, especially in harsh climates dust storms, the building construction of the respiratory system is more important. At present, China Northwest sandstorm weather is more and more frequent, people's daily life is largely influenced by such housing for sandstorms defense and improve indoor living environment is imminent, as a medium of contact with the outside of the building, balcony is the corresponding design strategy essential. In this paper, eco-balcony is designed to meet the climatic characteristics Northwest China just green balcony of many eco-energy saving means of a way of thinking. To achieve the man and nature, architecture and environment symbiotic green building, the local conditions must be taken into account this important factor in regional architectural design to the balcony, adhere to the principle can be implemented for the study, so the green balcony design technology can have popularization.

\section{Acknowledgements}

National Social Science Fund Project (Number: 213176).

\section{References}

[1] Yang Bo. Hot Summer and Cold Winter Area Multi-Storey Residential Climate Projections Balcony Adaptive Design Study [D]. Master's Thesis of Huazhong University of Science and Technology. 2004.

[2] Li Qiushi. Anti-dust and strategy of building ventilation in Northwest China[J]. Settlements research, 2014.01.

[3] Qinpei Liang, cold areas of green roof design method [D]. Dalian University of Technology master's thesis .2009

[4] Li Suyan. Building Energy Efficiency and Insulation Balcony Design [J]. Construction \& Engineering 2013. 05. 